\title{
On the origin of radio emission in Radio-Quiet AGN and their connection to X-rays
}

\author{
F. Panessa* \\ Istituto di Astrofisica Spaziale e Fisica Cosmica (IASF-INAF), via del Fosso del Cavaliere 100, \\ 00133 Roma, Italy \\ E-mail: francesca.panessa@iaps.inaf.it
}

L. Bassani

Istituto di Astrofisica Spaziale e Fisica Cosmica (IASF-INAF), Via P. Gobetti 101, 40129

Bologna, Italy

E-mail: bassanidiasfbo.inaf.it

\section{A. Bazzano}

Istituto di Astrofisica Spaziale e Fisica Cosmica (IASF-INAF), via del Fosso del Cavaliere 100, 00133 Roma, Italy

E-mail: angela.bazzano@iaps.inaf.it

\section{G. Bicknell}

Research School of Astronomy \& Astrophysics, Mt Stromlo Observatory, Cotter Rd., Weston, ACT 2611, Australia

E-mail: geoffemso.anu.edu.au

\section{P. Castangia}

Osservatorio Astronomico di Cagliari (OAC-INAF), Loc. Poggio dei Pini, Strada 54, 09012 Capoterra (CA), Italy

E-mail: pcastang@oa-cagliari.inaf.it

\section{Giroletti}

Istituto di Radioastronomia (IRA-INAF), Via P. Gobetti 101, 40129 Bologna, Italy

E-mail: girolettieira.inaf.it

\section{E. Maiorano}

Istituto di Astrofisica Spaziale e Fisica Cosmica (IASF-INAF), Via P. Gobetti 101, 40129 Bologna, Italy

E-mail: maioranodiasfbo.inaf.it

\section{A. Malizia}

Istituto di Astrofisica Spaziale e Fisica Cosmica (IASF-INAF), Via P. Gobetti 101, 40129 Bologna, Italy

E-mail: malizia@iasfbo.inaf.it

\section{A. Tarchi}

Osservatorio Astronomico di Cagliari (OAC-INAF), Loc. Poggio dei Pini, Strada 54, 09012

Capoterra (CA), Italy

E-mail: atarchi@oa-cagliari.inaf.it 


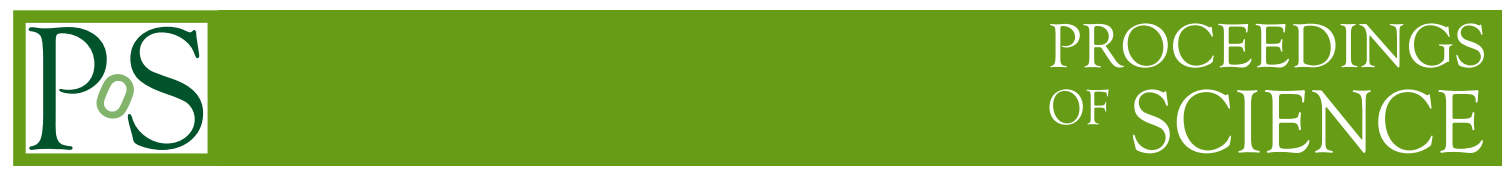

\section{P. Ubertini}

Istituto di Astrofisica Spaziale e Fisica Cosmica (IASF-INAF), via del Fosso del Cavaliere 100, 00133 Roma, Italy

E-mail: pietro.ubertini@iaps.inaf.it

The accretion-ejection mechanism acting in Active Galactic Nuclei (AGN) is one of the main astrophysical open issues, being connected to the role of AGN feedback in galaxy formation evolution studies. The X-ray emission in AGN, associated with the accretion flow, is strongly coupled with the radio emission, associated with a jet. Strong correlations between the radio and the X-ray luminosities are found both in radio-loud (RL) and in radio-quiet (RQ) AGN, despite the fact that in RQ AGN jets are often absent or very weak. For two well defined and complete samples of low and high luminosity AGN, we have investigated the origin of the radio emission. In particular, for the low luminosity AGN sample, I will present the results from the first census of VLBI sub-parsec cores of a complete sample of radio-quiet Seyfert galaxies. Interestingly, at milli arc-seconds scales the radio emission does not seem to correlate with X-rays anymore.

12th European VLBI Network Symposium and Users Meeting,

7-10 October 2014

Cagliari, Italy

${ }^{*}$ Speaker. 


\section{Introduction}

The connection between the radio emission (linked to the jet) and the X-ray emission (from accretion) is one of the key ingredients in AGN-galaxy evolutionary models (e.g., La Franca, Melini, Fiore et al. 2010). However, our knowledge on the relation between the accretion disk, the X-ray corona and the accelerated particles in jets is still poor and incomplete. From observations, we know that the radio and the X-ray emission are correlated in different classes of AGN (Canosa et al. 1999, Brinkmann et al. 2000, Panessa et al. 2007). Correlation slopes around 0.5-0.7 are typically interpreted within radiatively inefficient accretion flow accompanied by a relativistic jet, while values around 1.4-1.6 are consistent with radiatively efficient accretion (Dong et al. 2014, Coriat et. 2011, Merloni et al. 2003; Falcke et al. 2004).

In correlating radio and X-ray emission, physical scales are important. Hard X-ray and Xray emission originate in the innermost region of an AGN, at few Schwarzschild radii from the black hole. On the other hand, radio Very Large Array (VLA) images of local Seyfert galaxies map spatial scales of the order of tens of kpc while images taken with the Very Large Baseline Interferometry (VLBI) techniques can map milli arcsecond angular scales, which translates into sub-pc scales for local galaxies. Observing AGN with different radio arrays sheds light on different radio components placed at different distances from the black hole. Here we summarize the latest results on the radio properties of two well defined Seyfert samples, one selected at hard X-rays and observed with the NVSS and SUMSS survey and one optically selected and surveyed with the EVN.

\section{The radio versus $X$-ray correlations in INTEGRAL AGN}

We have selected a complete sample of hard X-ray AGN observed by INTEGRAL (Bird et al. 2010, Malizia et al. 2009) and we have analyzed the radio images from the NVSS and SUMSS surveys. We have detected a large fraction of radio sources (89\%), with different morphologies: thirty-six of the 70 objects (51\%) detected in the NVSS/SUMSS maps show an unresolved (22) or slightly resolved (14) central source coincident, within the uncertainties, with the position of the optical nucleus, 32 objects show a resolved morphology while two sources show an "ambiguous" morphology.

In figure 1 we plot the $1.4 \mathrm{GHz}$ peak luminosity versus the $20-100 \mathrm{keV}$ (left) and $2-10 \mathrm{keV}$ (right) luminosity with its best fit regression lines. The two correlations are both highly significant and display a very similar correlation slope, around 1-1.2, clearly steeper than the classical 0.6 value found in the fundamental plane for BH activity and consistent with the 1.4 expected for sources belonging to the efficient accretion branch (Coriat et al. 2011, Dong et al. 2014). This may imply that the INTEGRAL AGN are X-ray dominated (accretion dominated) sources accreting at high Eddington ratios and the high energy emission from the central engine is related to the radio emission averaged over kpc scales (Panessa et al. 2015).

\section{The EVN survey of local Seyfert galaxies}

For a complete optically selected sample of radio-quiet local Seyfert galaxies we have performed the first systematic study at VLBI spatial resolutions with the EVN, with the purpose of 

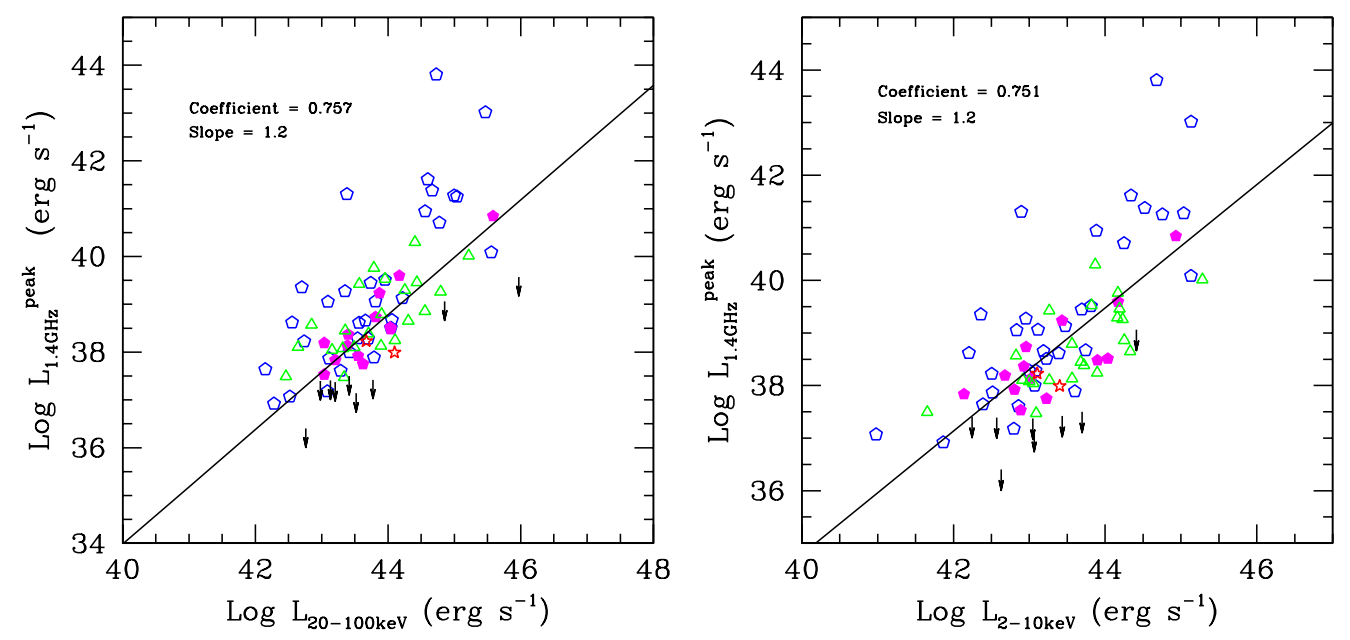

Figure 1: Left panel: $1.4 \mathrm{GHz}$ peak versus $20-100 \mathrm{keV}$ luminosity. Right panel: $1.4 \mathrm{GHz}$ peak versus 2-10 $\mathrm{keV}$ luminosity. The relative best fit regression line is shown for each correlation. Different morphological classes are marked as follows: resolved sources (R) are blue empty pentagons, slightly resolved (S) sources are magenta solid pentagons, unresolved (U) sources are green empty triangles, ambiguous (A) sources are red stars. Upper limits are marked as black arrows (see Panessa et al. 2015).

characterizing statistically the physical properties of the sub-pc cores and the incidence of associated jet/outflow structures.

We found that the milliarcsecond radio cores of Seyfert galaxies display a variety of morphologies, power and emission properties. The detection rate is around $60 \%$ (up to $\sim 74 \%$ ), at $5 \mathrm{GHz}$, which is significant considering the low radio powers here sampled. We find evidence or hints of the presence of a jet/outflow feature in the majority of the VLBI detected sources. In figure 2 (left panel) we show an example of an EVN image of one of the most interesting sources belonging to our sample, NGC 4051: a flat compact core is present plus a symmetric structure of two mini-lobes resembling the morphology of a mini radio-galaxy (Giroletti \& Panessa 2009).

In figure 2 (right panel) we show the $5 \mathrm{GHz}$ VLBI mas scales radio core luminosities versus the 2-10 keV unabsorbed luminosity. No significant correlation is found. This suggests that the $\mathrm{X}$-ray emission correlates more significantly with the tens of parsec and kpc scale radio emission rather than with the nuclear sub-pc scales or that the correlation holds at high luminosity regimes better than in LLAGN. It is known that the non simultaneity of the radio and X-ray observations in AGN correlations introduces a significant source of scatter, therefore, since there was no simultaneity in both studies, we expect the VLA and VLBI correlations to be affected in a similar way by flux variability.

\section{Acknowledgements}

F.P. acknowledges support by INTEGRAL ASI/INAF n. 2013-025.R.O. 

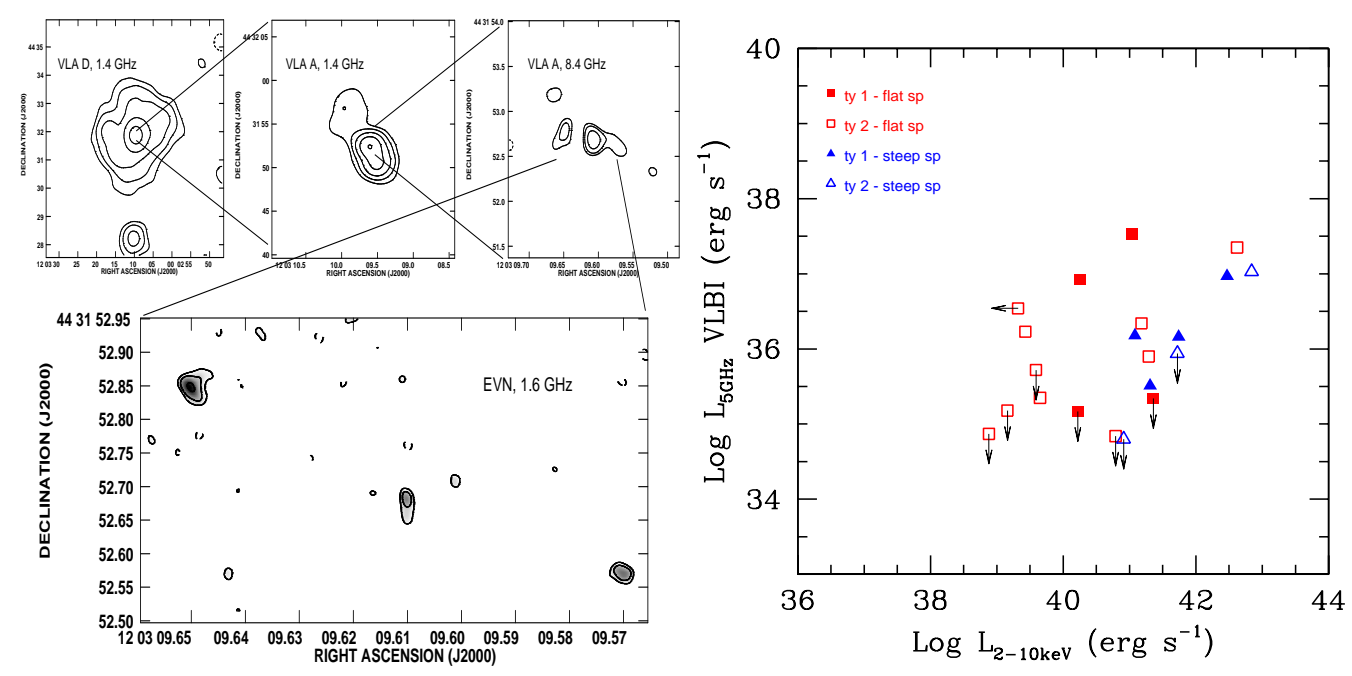

Figure 2: Left panel: VLA (top panels) and EVN images of NGC 4051 (Giroletti \& Panessa 2009). Right panel: VLBI radio luminosity at $5 \mathrm{GHz}$ versus 2-10 keV unabsorbed luminosity for a complete sample of optically selected Seyfert galaxies. Type 1 and type 2 Seyferts are indicated with filled and empty symbols, respectively; red squares show flat spectrum radio sources, blues triangles show steep spectrum ones (Panessa \& Giroletti 2013).

\section{References}

[1] Bird, A. J., et al. 2010, ApJS, 186, 1

[2] Bonchi, A., La Franca, F., Melini, G., Bongiorno, A., \& Fiore, F. 2013, MNRAS, 429, 1970

[3] Brinkmann, W., Laurent-Muehleisen, S. A., Voges, W., et al. 2000, A\&A, 356, 445

[4] Canosa, C. M., Worrall, D. M., Hardcastle, M. J., \& Birkinshaw, M. 1999, MNRAS, 310, 30

[5] Coriat M., et al., 2011, MNRAS, 414, 677

[6] Dong, A.-J., Wu, Q., \& Cao, X.-F. 2014, ApJL, 787, L20

[7] Falcke, H., Körding, E., \& Markoff, S. 2004, A\&A, 414, 895

[8] Giroletti, M., \& Panessa, F. 2009, ApJL, 706, L260

[9] La Franca, F., Melini, G., \& Fiore, F. 2010, ApJ, 718, 368

[10] Malizia, A., Stephen, J. B., Bassani, L., et al. 2009, MNRAS, 399, 944

[11] Merloni, A., Heinz, S., \& di Matteo, T. 2003, MNRAS, 345, 1057

[12] Panessa, F., Tarchi, A., Castangia, P., et al. 2015, MNRAS, 447, 1289

[13] Panessa, F., \& Giroletti, M. 2013, MNRAS, 432, 1138

[14] Panessa, F., Barcons, X., Bassani, L., Cappi, M., Carrera, F. J., Ho, L. C., \& Pellegrini, S. 2007, A\&A, 467,519 